\title{
Proper Use of Inflammatory Bowel Disease Drugs during Pregnancy
}

\author{
S.L. Kanis C.J. van der Woude \\ Department of Gastroenterology and Hepatology, Erasmus University Medical Center Rotterdam, Rotterdam, \\ The Netherlands
}

\section{Key Words}

Counseling · Fertility · Inflammatory bowel disease ·

Pregnancy · Treatment

\begin{abstract}
Crohn's disease and ulcerative colitis, referred to as inflammatory bowel disease (IBD), are chronic, relapsing conditions. Patients are often diagnosed at a reproductive age, and therefore questions about fertility and reproductions often arise. Preconceptional counseling is the most important aspect in the management of IBD patients with a pregnancy wish. Patients should be counseled on the influence of IBD and IBD drugs on pregnancy. Most drugs are not related to adverse outcome while used during pregnancy. Active disease is related to adverse outcomes; therefore, it is of utmost importance to strive for remission before conception and during pregnancy.

(c) 2016 The Author(s)

Published by S. Karger AG, Basel
\end{abstract}

\section{Introduction}

Crohn's disease (CD) and ulcerative colitis (UC), referred to as inflammatory bowel disease (IBD), are chronic, relapsing conditions. IBD typically arises at a reproductive age and because the maternal age during first preg-

\section{KARGER \\ E-Mail karger@karger.com} www.karger.com/ddi

\section{(c) 2016 The Author(s) \\ Published by S. Karger AG, Basel 0257-2753/16/0345-0061\$39.50/0}

This article is licensed under the Creative Commons AttributionNonCommercial-NoDerivatives 4.0 International License (CC BYNC-ND) (http://www.karger.com/Services/OpenAccessLicense). Usage and distribution for commercial purposes as well as any distribution of modified material requires written permission. nancy is increasing, an increasing number of IBD women will become pregnant after diagnosis. Although there are still misbeliefs with regard to pregnancy and IBD, it has been generally accepted that IBD or its treatment is no reason to advice against pregnancy. The most important risk factor for adverse pregnancy outcomes is the presence of disease activity. Disease activity at time of conception and during pregnancy is associated with a higher rate of spontaneous abortion, preterm delivery, thromboembolic events, emergency caesarean section and low birth weight, whereas the majority of pregnancies in women with quiescent IBD are uncomplicated. This indicates the necessity to maintain remission with the continuation of medication. Therefore, knowledge on the effects of IBD and IBD drugs on the child in utero is of utmost importance for IBD patients with a pregnancy wish.

\section{Counseling IBD Patients with a Child Wish}

Patients should be counseled on the influence of IBD and IBD drugs on pregnancy. Life style advise such as the necessity to stop smoking and the use of folic acid before conception should also be discussed. In general, it is advised to strive for disease remission at least 6 months before conception, since disease activity negatively influences fertility and pregnancy outcome. 
IBD patients have fewer children compared to the general population $[1,2]$. Incorrect beliefs and poor knowledge on IBD and pregnancy probably contribute to the high rate of voluntary childlessness within the IBD population, resulting in a lower average number of offspring $[3,4]$. Recent studies show that fertility is not influenced by the presence of UC or CD $[5,6]$. However, active disease is related to subfertility in both male and female patients [7]. Possible reasons are inflammation of the colon involving the fallopian tubes and ovaries, poor nutrition, depression, decreased libido and dyspareunia caused by perianal disease [8]. Female UC patients who underwent a colectomy with an ileal pouch anal anastomosis (IPAA) have a 3-fold increased risk of subfertility than female UC patients who did not [9-11]. The reason for subfertility after IPAA surgery is most likely destruction of fimbria, the higher rate of hydrosalpinx and tubal obstruction following pelvic surgery [12]. Recently, 2 small retrospective studies showed that infertility rates after laparoscopic IPAA surgery are lower than after open IPAA surgery [13, 14]. This might be explained by a reduced adhesion formation after laparoscopic surgery. Males with UC who had IPAA surgery may experience erectile dysfunction and retrograde ejaculation. However, studies show either no change or an improvement in sexual function after surgery in male patients $[15,16]$.

\section{IBD Drugs and Fertility in Male IBD Patients}

Sulfasalazine causes a reversible, dose-related, decrease in sperm count and motility in male patients $[17,18]$. As a result, male patients using sulfasalazine are less fertile. Sulfasalazine should be switched to a different 5-ASA drug in case of a reproduction wish.

Corticosteroids can cause a reversible decrease in sperm motility and concentration [19]. However, there seems to be no link between steroid use and infertility [20, 21]. Methotrexate (MTX) causes oligospermia, which improves within a few months after stopping [22]. Moreover, MTX is teratogenic and contraindicated in both men and women wishing to conceive. It is advised to stop MTX3-6 months before conception. Azathioprine (AZA) does not reduce semen quality and therefore fertility in male IBD patients [23]. A large prospective study including 115 pregnancies fathered by men using thiopurines (AZA/6-mercaptopurine (6-MP)) during conception showed no increased rate in major congenital anomalies [24]. Also, a meta-analysis published in 2013 showed no association between congenital abnormalities and the use of thiopurines by the father at the time of conception [25]. Therefore, it is not recommended that male IBD patients stop thiopurine treatment before conception. The effect of anti-TNF on male fertility has not been extensively examined. Infliximab (IFX) seems to affect semen quality by reducing motility [66], but data are conflicting as men with spondyloarthropathies tended to have better sperm qualities when receiving anti-TNF therapy [26]. Few studies investigated the effects of male IFX use during conception upon the fetus, but these studies found no evidence of an increased risk of adverse birth outcome [27, 28]. Therefore, male IBD patients should not be advised to stop IFX treatment before conception. There have been no studies on the effect of adalimumab on male fertility.

\section{5-ASA during Conception and Pregnancy in Women}

5-ASA drugs are considered low-risk drugs during pregnancy. However, formulations containing dibutyl phthalate coating are re-categorized from FDA category $B$ (animal reproduction studies have failed to demonstrate a risk to the fetus and there are no adequate and well-controlled studies in pregnant women) to C (animal reproduction studies have shown an adverse effect on the fetus and there are no adequate and well-controlled studies in humans, but potential benefits may warrant use of the drug in pregnant women despite potential risks) because animal studies have shown an increased risk of congenital defects in the male urogenital tract [30]. Furthermore, high-dose dibutyl phthalate exposure is possibly associated with precocious puberty [31-33]. Therefore, formulations containing dibutyl phthalate coating should be avoided if possible. Sulfasalazine interferes with the folate metabolism; therefore, $2 \mathrm{mg} /$ day of folic acid supplementation is recommended in women contemplating pregnancy.

\section{IBD Medication: Thiopurines during Conception and Pregnancy in Women}

During pregnancy, the active metabolite 6-thioguanine crosses the placenta but the prodrugs AZA and 6-MP do not [34]. Recent studies showed no increased risk of adverse pregnancy outcome in the case of thiopurine use during pregnancy [35-37]. This is in contrast with earlier published studies that described adverse pregnancy outcomes in cases of thiopurine use [38,39]. However, these earlier studies failed to take in to account the disease activ-
Kanis/van der Woude 
ity during pregnancy, as it is well known that disease activity during pregnancy increases the risk of preterm delivery and low birth weight. It is therefore recommended that thiopurine should be continued during pregnancy as the risk of active disease outweighs the risks associated with thiopurine use. With respect to growth and development in children exposed to thiopurines in utero, a normal growth and development was reported in children followed up to 6 years of age [40]. Furthermore, the ongoing prospective PIANO registry did not observe an increased risk of congenital anomalies or pregnancy complications among 337 children who were exposed to a thiopurine during pregnancy [41]. In case of longstanding remission using combination therapy with an anti-TNF agent, it may be considered to stop the thiopurine before conception. However, patient's medication history and disease severity should be taken into account. Additionally, thiopurines are not recommended to be started during pregnancy because of the relatively late disease response and risks of bone marrow suppression and pancreatitis.

\section{Methotrexate}

MTX and thalidomide are teratogenic $[42,43]$ and (barrier) contraceptive methods are strongly recommended during therapy. Patients treated with MTX who express a pregnancy wish should discontinue MTX 6 months prior to contraceptive cessation. Alternative maintenance therapy with a low-risk profile during pregnancy should be started. Patients should be carefully monitored for any recurrence of disease activity with the new medication regimen. If however unplanned conception occurs during MTX treatment, it needs to be stopped immediately and high-dose folate replacement should be started. In addition, patients should be referred to a specialized obstetrician to discuss the effects of MTX on the child.

\section{Anti-TNF Agents}

IFX and ADA are both IgG1 antibodies capable of crossing the placenta in the second and third trimester of pregnancy [44]. Drug levels in infants exceed maternal anti-TNF levels and are dependent on the timing of antiTNF cessation during pregnancy [45]. A systematic review showed that anti-TNF therapy does not increase the risk of unfavorable pregnancy outcomes among women with IBD [46]. The long-term effects of in utero exposure to anti-TNF are not extensively explored. A recent study shows a normal health outcome and first year development in children exposed to anti-TNF, comparable to children of non-IBD controls [47]. Also preliminary re- sults of 2 ongoing studies showed normal growth and development in anti-TNF exposed children in the first few years of life $[48,49]$. It should however be mentioned that combination therapy with immunomodulators did increase the risk of infections in offspring in the ongoing PIANO registry. In addition, continuing anti-TNF during pregnancy may have consequences for the vaccination program of the child because live vaccines should be deferred until anti-TNF levels are undetectable in the child. Fetal exposure to high levels of anti-TNF treatment may be avoided by stopping anti-TNF treatment at the end of the second trimester if patients are in sustained remission. A prospective study, comprising 83 pregnancies exposed to anti-TNF, showed that early discontinuation before gestational week 25 did not increase the risk of a flare and resulted in significantly lower levels of antiTNF in cord blood [50]. Certolizumab pegol (CZP) is a PEGylated $\mathrm{Fab}^{\prime}$ fragment of a humanized anti-TNFa monoclonal antibody. This Fab' fragment crosses the placenta by passive diffusion and not by active transfer like IFX and ADA. Therefore, the drug levels reaching the fetus are probably lower. A recent study analyzed the pregnancy outcomes of in utero CZP exposure, suggesting a lack of harmful effect of maternal CZP exposure [51].

Golimumab is a fully humanized monoclonal antibody, very similar to ADA. There is limited data on pregnancy outcomes when used during pregnancy, although the safety profile is probably similar to the other anti-TNF drugs.

Data on anti-TNF initiation during pregnancy remain scarce, although several successful cases have been reported $[52,53]$. In specific cases, it could be considered to start an anti-TNF agent in the case of steroid-refractory disease during pregnancy, as it is known that active disease cause maternal and fetal risks.

\section{Vedolizumab}

Data on the outcome of children exposed in utero to vedolizumab are still limited. As vedolizumab is also an IgG1 antibody, this drug is likely to be transferred through the placenta during the second and third trimester of pregnancy. A recent report showed the outcomes of 24 women exposed to vedolizumab, which resulted in 11 live births [54]. No teratogenicity was reported in animals exposed to high doses of vedolizumab [55]. Because vedolizumab is gut specific, a hypothetical concern might be an increased risk of gastrointestinal infections, such as the rotavirus in the newborn. However, more results are needed to adequately counsel patients using vedolizumab. 
Treatment of a Disease Relapse during Pregnancy

Corticosteroids, particularly prednisolone, may be necessary for induction of remission during pregnancy. Before initiating new drugs during pregnancy, it is of importance to estimate the severity and extensiveness of the disease activity. However, clinical and laboratory results may fail to prove real active disease and fecal calprotectin is not validated during pregnancy. Therefore, performing a lower endoscopy might be warranted. Recently, a systematic review concluded that lower endoscopy is of low risk for mother and child in all 3 trimesters of pregnancy [56]. Additionally, a prospective study comprising 42 pregnant women who underwent 47 lower endoscopies during pregnancy showed no adverse outcome related to the endoscopy in all 3 trimesters [57]. Therefore, lower endoscopy can be performed during all trimesters when strongly indicated. With respect to the treatment of a relapse during pregnancy with corticosteroids, recent data from a large population-based cohort showed no increased risk of malformations [58]. Although an earlier meta-analysis showed an increase in children with a cleft palate [59], it seems that using corticosteroids during pregnancy is of low risk. There have been reports of neonatal adrenal suppression following exposure to corticosteroids in the late third trimester. Therefore, a pediatrician should carefully examine neonates exposed to corticosteroids in utero after birth. Furthermore, corticosteroids are known to increase the risk of gestational diabetes and hypertension, and pregnant women should be followed up stringently by a gynecologist [60]. Prednisolone is the preferred corticosteroid during pregnancy, because of the limited transplacental passage $[61,62]$. No ad- verse pregnancy outcomes following budesonide use during pregnancy has been reported [63] and should be considered alongside treatment with prednisolone. Dexamethasone, betamethasone and hydrocortisone are best avoided. Other drugs that might be initiated for the treatment of a relapse during pregnancy include the above-mentioned drugs, mesalazine and anti-TNF. Cyclosporine-A treatment is used as rescue therapy for severe, refractory UC. Data on safety profile during pregnancy mainly come from organ transplant patients who became pregnant. A meta-analysis assessing the use of cyclosporine-A during pregnancy showed no adverse pregnancy outcomes [64]. Small case series in UC women with severe disease activity during pregnancy who were treated with cyclosporine-A showed no increased risk of adverse pregnancy outcomes or malformations [65].

\section{Conclusion}

Most drugs are not related to adverse outcome while used during pregnancy. Active disease is related to adverse outcomes; therefore, it is of utmost importance to strive for remission before conception and during pregnancy. Because knowledge on pregnancy, drugs and IBD seems limited in IBD patients, counseling of patients who are planning pregnancy is advised before conception.

\section{Disclosure Statement}

No disclosure.

\section{References}

1 Marri SR, Ahn C, Buchman AL: Voluntary childlessness is increased in women with inflammatory bowel disease. Inflamm Bowel Dis 2007;13:591-599.

2 Mountifield R, et al: Fear and fertility in inflammatory bowel disease: a mismatch of perception and reality affects family planning decisions. Inflamm Bowel Dis 2009;15:720-725.

3 Manosa M, et al: Fecundity, pregnancy outcomes, and breastfeeding in patients with inflammatory bowel disease: a large cohort survey. Scand J Gastroenterol 2013;48:427-432.

4 Selinger CP, et al: Inflammatory bowel disease and pregnancy: lack of knowledge is associated with negative views. J Crohns Colitis 2013;7:e206-e213.
5 Baird DD, Narendranathan M, Sandler RS: Increased risk of preterm birth for women with inflammatory bowel disease. Gastroenterology 1990;99:987-994.

6 Hudson M, et al: Fertility and pregnancy in inflammatory bowel disease. Int J Gynaecol Obstet 1997; 58:229-237.

7 Riis L, et al: Does pregnancy change the disease course? A study in a European cohort of patients with inflammatory bowel disease. Am J Gastroenterol 2006;101:1539-1545.

8 Kane SV: Inflammatory bowel disease, women, and pregnancy. Gastroenterol Hepatol (N Y) 2013;9:741-743.

9 Waljee A, et al: Threefold increased risk of infertility: a meta-analysis of infertility after il- eal pouch anal anastomosis in ulcerative colitis. Gut 2006;55:1575-1580.

10 Rajaratnam SG, et al: Impact of ileal pouchanal anastomosis on female fertility: metaanalysis and systematic review. Int J Colorectal Dis 2011;26:1365-1374.

11 Cornish JA, et al: The effect of restorative proctocolectomy on sexual function, urinary function, fertility, pregnancy and delivery: a systematic review. Dis Colon Rectum 2007; 50:1128-1138

12 van der Woude CJ, et al: The second European evidenced-based consensus on reproduction and pregnancy in inflammatory bowel disease. J Crohns Colitis 2015;9:107124 
13 Beyer-Berjot L, et al: A total laparoscopic approach reduces the infertility rate after ileal pouch-anal anastomosis: a 2-center study. Ann Surg 2013;258:275-282.

14 Bartels SA, et al: Significantly increased pregnancy rates after laparoscopic restorative proctocolectomy: a cross-sectional study. Ann Surg 2012;256:1045-1048.

15 Johnson E, et al: Morbidity and functional outcome after restorative proctocolectomy for ulcerative colitis. Eur J Surg 2001;167:4045.

16 Davies RJ, et al: A prospective evaluation of sexual function and quality of life after ileal pouch-anal anastomosis. Dis Colon Rectum 2008;51:1032-1035.

17 Toth A: Reversible toxic effect of salicylazosulfapyridine on semen quality. Fertil Steril 1979;31:538-540.

18 Birnie GG, McLeod TI, Watkinson G: Incidence of sulphasalazine-induced male infertility. Gut 1981;22:452-455.

19 Heetun ZS, et al: Review article: reproduction in the patient with inflammatory bowel disease. Aliment Pharmacol Ther 2007;26:513533.

20 Narendranathan $M$, et al: Male infertility in inflammatory bowel disease. J Clin Gastroenterol 1989;11:403-406.

21 Feagins LA, Kane SV: Sexual and reproductive issues for men with inflammatory bowel disease. Am J Gastroenterol 2009;104:768773.

22 Sussman A, Leonard JM: Psoriasis, methotrexate, and oligospermia. Arch Dermatol 1980;116:215-217.

23 Dejaco C, et al: Azathioprine treatment and male fertility in inflammatory bowel disease. Gastroenterology 2001;121:1048-1053.

24 Hoeltzenbein M, et al: Pregnancy outcome after paternal exposure to azathioprine/6-mercaptopurine. Reprod Toxicol 2012;34:364369 .

25 Akbari M, et al: Systematic review and metaanalysis on the effects of thiopurines on birth outcomes from female and male patients with inflammatory bowel disease. Inflamm Bowel Dis 2013;19:15-22.

26 Villiger PM, et al: Effects of TNF antagonists on sperm characteristics in patients with spondyloarthritis. Ann Rheum Dis 2010;69: 1842-1844.

27 Paschou S, et al: Fertility and reproduction in male patients with ankylosing spondylitis treated with infliximab. J Rheumatol 2009;36: 351-354.

28 Puchner R, et al: Impact of TNF-blocking agents on male sperm characteristics and pregnancy outcomes in fathers exposed to TNF-blocking agents at time of conception. Clin Exp Rheumatol 2012;30:765-767.

29 Sands K, et al: Review article: the safety of therapeutic drugs in male inflammatory bowel disease patients wishing to conceive. Aliment Pharmacol Ther 2015;41:821-834

30 Hernandez-Diaz S, Su YC, Mitchell AA, Kelley KE, Calafat AM, Hauser R: Medications as a potential source of exposure to phthalates among women of childbearing age. Reprod Toxicol 2013;37:1-5.

31 Jurewicz J, Hanke W: Exposure to phthalates: reproductive outcome and children health. A review of epidemiological studies. Int J Occup Med Environ Health 2011;24:115-141.

32 Norgard B, Czeizel AE, Rockenbauer M, Olsen J, Sorensen HT: Population-based case control study of the safety of sulfasalazine use during pregnancy. Aliment Pharmacol Ther 2001;15:483-486.

33 Rahimi R, Nikfar S, Rezaie A, Abdollahi M: Pregnancy outcome in women with inflammatory bowel disease following exposure to 5-aminosalicylic acid drugs: a meta-analysis. Reprod Toxicol 2008;25:271-275.

34 Saarikoski S, Seppala M: Immunosuppression during pregnancy: transmission of azathioprine and its metabolites from the mother to the fetus. Am J Obstet Gynecol 1973;115: 1100-1106.

35 Casanova MJ, et al: Safety of thiopurines and anti-TNF- $\alpha$ drugs during pregnancy in patients with inflammatory bowel disease. Am J Gastroenterol 2013;108:433-440.

36 Coelho J, et al: Pregnancy outcome in patients with inflammatory bowel disease treated with thiopurines: cohort from the CESAME study. Gut 2011;60:198-203.

37 Shim L, et al: The effects of azathioprine on birth outcomes in women with inflammatory bowel disease (IBD). J Crohns Colitis 2011;5: 234-238.

38 Cleary BJ, Kallen B: Early pregnancy azathioprine use and pregnancy outcomes. Birth Defects Res A Clin Mol Teratol 2009;85:647654.

39 Norgard B, et al: Azathioprine, mercaptopurine and birth outcome: a population-based cohort study. Aliment Pharmacol Ther 2003; $17: 827-834$

40 de Meij TG, et al: Long-term follow-up of children exposed intrauterine to maternal thiopurine therapy during pregnancy in females with inflammatory bowel disease. Aliment Pharmacol Ther 2013;38:38-43.

41 McConnell RA, Mahadevan U: Use of immunomodulators and biologics before, during, and after pregnancy. Inflamm Bowel Dis 2016;22:213-223

42 Kozlowski RD, Steinbrunner JV, MacKenzie AH, Clough JD, Wilke WS, Segal AM: Outcome of first-trimester exposure to low-dose methotrexate in eight patients with rheumatic disease. Am J Med 1990;88:589-592.

43 Smithells RW, Newman CG: Recognition of thalidomide defects. J Med Genet 1992;29: 716-723.

44 Kane SV, Acquah LA: Placental transport of immunoglobulins: a clinical review for gastroenterologists who prescribe therapeutic monoclonal antibodies to women during conception and pregnancy. Am J Gastroenterol 2009; 104:228-233.

45 Zelinkova Z, et al: High intra-uterine exposure to infliximab following maternal anti-
TNF treatment during pregnancy. Aliment Pharmacol Ther 2011;33:1053-1058.

46 Zelinkova Z, et al: Effects of discontinuing anti-tumor necrosis factor therapy during pregnancy on the course of inflammatory bowel disease and neonatal exposure. Clin Gastroenterol Hepatol 2013;11:318-321.

47 Narula N, et al: Anti-TNFa therapies are safe during pregnancy in women with inflammatory bowel disease: a systematic review and meta-analysis. Inflamm Bowel Dis 2014;20 1862-1869.

48 Kanis S, de Lima A, Zelinkova Z, Dijkstra G, West R, Ouwendijk R, de Boer N, Pierik M, van der Meulen-De Jong A, Oostenbrug $L$, Romberg-Camps M, Bodelier A, Oldenburg B, Hoentjen F, Beukers R, Jansen J, van der Woude CJ: Long term outcome of children born to IBD mothers: preliminary result from a multicenter retrospective study in the Netherlands. Clin Ther Observ 2015, P507.

49 Mahadevan U, Martin CF, Sandler RS, et al: PIANO: a 1000 patient prospective registry of pregnancy outcomes in women with IBD exposed to immunomodulators and biologic therapy. Gastroenterology 2012; 142(5 suppl 1):S149.

50 de Lima A, et al: Tailored anti-TNF therapy during pregnancy in patients with IBD: maternal and fetal safety. Gut 2015;pii:gutjnl2015-309321.

51 Clowse ME, et al: Pregnancy outcomes in subjects exposed to certolizumab pegol. J Rheumatol 2015;42:2270-2278.

52 Aratari $\mathrm{A}$, et al: Intentional infliximab use during pregnancy for severe steroid-refractory ulcerative colitis. J Crohns Colitis 2011;5: 262.

53 Schnitzler F, et al: Outcome of pregnancy in women with inflammatory bowel disease treated with antitumor necrosis factor therapy. Inflamm Bowel Dis 2011;17:18461854.

54 Dubinsky M, Mahadevan U, Vermeire S, Abhyankar B, Lasch K: P563. Vedolizumab exposure in pregnancy: outcomes from clinical studies in inflammatory bowel disease. J Crohns Colitis 2015;9:S361-S362.

55 Entyvio [package insert]. Deerfield, Takeda Pharmaceuticals America, Inc., 2014.

56 De Lima A, Galjart B, Wisse PH, Bramer WM, van der Woude CJ: Does lower gastrointestinal endoscopy during pregnancy pose a risk for mother and child? - a systematic review. BMC Gastroenterol 2015;15:15.

57 De Lima A, Zelinkova Z, van der Woude CJ: A prospective study of the safety of lower gastrointestinal endoscopy during pregnancy in patients with inflammatory bowel disease. J Crohns Colitis 2015;9:519-524.

58 Park-Wyllie L, Mazzotta P, Pastuszak A, Moretti ME, Beique L, Hunnisett L, et al: Birth defects after maternal exposure to corticosteroids: prospective cohort study and metaanalysis of epidemiological studies. Teratology 2000;62:385-392. 
59 Hviid A, Molgaard-Nielsen D: Corticosteroid use during pregnancy and risk of orofacial clefts. CMAJ 2011;183:796-804.

60 Homar V, Grosek S, Battelino T: High-dose methylprednisolone in a pregnant woman with Crohn's disease and adrenal suppression in her newborn. Neonatology 2008;94:306309.

61 van Runnard Heimel PJ, Franx A, Schobben AF, Huisjes AJ, Derks JB, Bruinse HW: Corticosteroids, pregnancy, and HELLP syndrome: a review. Obstet Gynecol Surv 2005; 60:57-70; quiz 73-74.
62 van Runnard Heimel PJ, Schobben AF, Huisjes AJ, Franx A, Bruinse HW: The transplacental passage of prednisolone in pregnancies complicated by early-onset HELLP syndrome. Placenta 2005;26:842845.

63 Beaulieu DB, Ananthakrishnan AN, Issa M, Rosenbaum L, Skaros S, Newcomer JR, et al: Budesonide induction and maintenance therapy for Crohn's disease during pregnancy. Inflamm Bowel Dis 2009;15:25-28.
64 Bar Oz B, Hackman R, Einarson T, Koren G: Pregnancy outcome after cyclosporine therapy during pregnancy: a meta-analysis. Transplantation 2001;71:1051-1055.

65 Branche J, Cortot A, Bourreille A, Coffin B, de Vos M, de Saussure P, et al: Cyclosporine treatment of steroid-refractory ulcerative colitis during pregnancy. Inflamm Bowel Dis 2009; 15:1044-1048.

66 Mahadevan U, et al: Infliximab and semen quality in men with inflammatory bowel disease. Inflamm Bowel Dis 2005;14:395-399. 